\title{
Fixed point theorems for contractive mappings and Ćirić-Maiti-Pal orbit mappings of contractive type in re-defined generalized metric spaces
}

\author{
Xiaoming Fan ${ }^{\mathrm{a}}$, Zhigang Wang ${ }^{\mathrm{b}, *}$ \\ ${ }^{a}$ College of Teacher Education, Harbin Normal University, Harbin, 150025, P. R. China. \\ ${ }^{b}$ School of Mathematical Sciences, Harbin Normal University, Harbin, 150025, P. R. China. \\ Communicated by P. Kumam
}

\begin{abstract}
In this paper, the re-defined generalized metric space which is equivalent to the generalized metric spaces defined by Jleli and Samet is presented so that some well-known spaces are incorporated in the area of re-defined generalized metric spaces. Some fixed point existence and uniqueness results of contractive and generalized contraction mappings defined on such metric spaces are provided. Especially, we discussed the fixed point existence results of Ćirić-Maiti-Pal orbit mappings of contractive type in the re-defined generalized metric spaces. In addition, some examples are provided to better support the fixed point results. (c)2017 All rights reserved.
\end{abstract}

Keywords: Re-defined generalized metric space, fixed point theorems, Ćirić-Maiti-Pal orbit mapping of contractive type, f-orbitally complete.

2010 MSC: 47H09, 54H 25.

\section{Introduction}

Fixed point theory is a very powerful tool and has been widely studied by many mathematicians and practitioners of nonlinear phenomena. There exist many different branches in the theory of fixed points, which are full developed today. During the past several decades, classical Banach contraction principle has been generalized in many directions, many mathematicians have given significant contributions to these areas. Ćirić introduced the notion of quasi-contraction mapping [4], Rhoades gave a comparative study for some generalized mappings of contractive type [23], Nieto proved some fixed point theorems in partially ordered sets in term of the non-decreasing mappings, non-increasing mappings as well as nonmonotone mappings, which are extension of the Banach contractive mapping $[18,19]$, much of their work has a profound influence on follow-up studies of other people $[5,6,18,19,22,24]$. As a generalization of the notion of metric space, Czerwik presented the notion of b-metric space [7]. Subsequently, a lot more people do work around this kind of spaces [11]. Hitzler and Seda have focused fixed point theorems on dislocated metric spaces defined by themselves [10], more relevant results based on such spaces followed

\footnotetext{
*Corresponding author

Email addresses: fanxm093@163.com (Xiaoming Fan), wangzg2003205@163.com (Zhigang Wang)
} 
in recent years [21, 28]. A lot of mathematicians [1, 2, 13-16, 27] have given fixed point results on modular spaces since Nakano [17] introduced the notion of modular spaces as a generalization of metric spaces in 1950. The second author et al. defined the class of $(\alpha, \psi)$-Meir-Keeler-Khan multivalued mappings recently and fixed point theorems and endpoints theorems are established on such mappings [25]. The first author also introduced the concepts of qpb-cyclic-Banach contraction mapping, qpb-cyclic-Kannan mapping and qpb-cyclic-quasi-contraction mapping and established the existence and uniqueness of fixed point theorems for these mappings in quasi-partial b-metric spaces [8]. Jleli and Samet [12] introduced the definition of the generalized metric spaces on which some spaces mentioned above have been unified based, that is, all of the standard metric spaces, the dislocated metric spaces, and the modular metric spaces $D_{\mathfrak{m}}$, which $\mathfrak{m}$ are with Fatou property, are generalized metric space.

In this paper, inspired by [12], the definition re-defined generalized metric space, an equivalent space of the generalized metric space defined by Jleli and Samet, is presented so that some spaces mentioned above belong to the domain of new definition, both contractive mappings and Ćirić-Maiti-Pal orbit mappings of contractive type defined in the re-defined generalized metric spaces are discussed in detailed. Some fixed point results for the two kinds of mappings are demonstrated in setting of the re-defined generalized metric spaces. Some examples are provided to verify the effectiveness of the results.

\section{The relationships between re-defined generalized metric spaces and other spaces}

\subsection{The re-defined generalized metric spaces}

Jleli and Samet introduced the notion of generalized metric space which is shown as follows.

Definition 2.1 ([12]). A generalized metric on a nonempty set $X$ is a function $D: X \times X \rightarrow[0,+\infty)$, satisfying the following conditions:

$\left(D_{1}\right)$ If $D(x, y)=0$, then $x=y$ for every $(x, y) \in X \times X$.

$\left(D_{2}\right) D(x, y)=D(y, x)$ for every $(x, y) \in X \times X$.

$\left(D_{3}\right)$ There exists $C>0$ such that if $\left\{x_{n}\right\}_{n \in \mathbb{N}} \in C(D, X, x)$, then $D(x, y) \leqslant C \limsup _{n \rightarrow \infty} D\left(x_{n}, y\right)$ for all $(x, y) \in X \times X$, where

$$
C(D, X, x)=\left\{\left\{x_{n}\right\} \subset X: \lim _{n \rightarrow \infty} D\left(x_{n}, x\right)=0\right\} .
$$

The pair $(X, D)$ is called a generalized metric space.

Let $X$ be a nonempty set and $D_{g}: X \times X \rightarrow[0,+\infty)$ be a given mapping. For every $x \in X$, let us define the set

$$
\mathscr{C}\left(D_{g}, X, x, 0\right)=\left\{\left\{x_{n}\right\} \subset X: \lim _{n \rightarrow \infty} D_{g}\left(x_{n}, x\right)=0\right\} .
$$

Definition 2.2. A re-defined generalized metric on a nonempty set $X$ is a function $D_{g}: X \times X \rightarrow[0,+\infty)$, satisfying the following conditions:

$\left(R_{G M}\right)$ If $D_{g}(x, y)=0$, then $x=y$ for every $(x, y) \in X \times X$.

$\left(R_{G}\right) D_{g}(x, y)=D_{g}(y, x)$ for every $(x, y) \in X \times X$.

$\left(R_{G}\right)$ There exists $C>0$ such that if $\left\{x_{n}\right\}_{n \in \mathbb{N}} \in \mathscr{C}\left(D_{g}, X, x, 0\right)$, then $D_{g}(x, y) \leqslant C \varphi\left(\limsup D_{g}\left(x_{n}, y\right)\right)$,

for all $(x, y) \in X \times X$, where $\varphi:[0,+\infty) \rightarrow(0,+\infty)$ is a continuous and monotone non-decreasing function satisfying $\varphi(0)=0$ and $\varphi(t) \geqslant t$ for all $t \geqslant 0$ and there exists an $M \in(1,+\infty)$ such that $\varphi(t) \leqslant M t$, for all $t>0$, here we call $M$ an associated number of $\varphi$.

A re-defined generalized metric space (RGMS) is a pair $\left(X, D_{g}, C, \varphi\right)$ such that $X$ is a nonempty set and $\mathrm{D}_{\mathrm{g}}$ is a re-defined generalized metric on $\mathrm{X}$. 
Remark 2.3. In Definition 2.2, it would not be difficult to find function $\varphi$ satisfying the conditions of $\left(\mathrm{RGM}_{3}\right)$. For example, the function

$$
\varphi(t)= \begin{cases}e^{t}-1, & t \in[0,1) \\ (e-1) t, & t \in[1,+\infty)\end{cases}
$$

$M \geqslant e-1$ can be taken as the associated number of $\varphi$.

Proposition 2.4. Any generalized metric $\mathrm{D}$ on $\mathrm{X}$ is equivalent to re-defined generalized metric $\mathrm{D}_{\mathrm{g}}$ on $\mathrm{X}$.

Proof. Let $\mathrm{D}$ be a generalized metric on $\mathrm{X}$. To verify the proposition, we only need to check that $\left(\mathrm{D}_{3}\right)$ is equivalent to the property $\left(\mathrm{RGM}_{3}\right)$. Indeed, if $\left(\mathrm{RGM}_{3}\right)$ holds, then for $\left\{x_{n}\right\}_{n \in \mathbb{N}} \in \mathscr{C}(\mathrm{D}, \mathrm{X}, \mathrm{x}, 0)$ and $(x, y) \in X \times X$, we get (since $\varphi(t) \leqslant M t)$

$$
\begin{aligned}
D_{g}(x, y) & \leqslant C \limsup _{n \rightarrow \infty} D_{g}\left(x_{n}, y\right) \\
& \leqslant C M \varphi\left(\limsup _{n \rightarrow \infty} D_{g}\left(x_{n}, y\right)\right) \\
& =C_{1} \varphi\left(\limsup _{n \rightarrow \infty} D_{g}\left(x_{n}, y\right)\right)
\end{aligned}
$$

where $C_{1}=C M$, therefore, $\left(D_{3}\right)$ holds. On the other hand, by taking $\varphi(t)=t$, the contrary is clear. This completes the proof.

Next we define convergent sequence, Cauchy sequence, and complete space in a natural way.

Definition 2.5. Let $\left(X, D_{g}, C, \varphi\right)$ be a re-defined generalized metric space, $\left\{x_{n}\right\}_{n} \in \mathbb{N}$ be a sequence in $X$ and $x \in X$. We say that $\left\{x_{n}\right\}_{\mathfrak{n} \in \mathbb{N}} D_{g}$-converges to $x$, if

$$
\left\{x_{n}\right\}_{n \in \mathbb{N}} \in \mathscr{C}\left(D_{g}, X, x, 0\right)
$$

We denote simply $D_{g}$-converges to $x$ by $x_{n} \stackrel{D_{g}}{\longrightarrow} x$.

Proposition 2.6. Let $\left(X, D_{g}\right)$ be a re-defined generalized metric space. Let $\left\{x_{n}\right\}$ be a sequence in $X$ and $(x, y) \in$ $\mathrm{X} \times \mathrm{X}$. If $\left\{\mathrm{x}_{\mathrm{n}}\right\} \mathrm{D}_{\mathrm{g}}$-converges to $\mathrm{x}$ and $\left\{\mathrm{x}_{\mathrm{n}}\right\} \mathrm{D}_{\mathrm{g}}$-converges to $\mathrm{y}$, then $\mathrm{x}=\mathrm{y}$.

Proof. Using the property $\left(\mathrm{RGM}_{3}\right)$, we have

$$
\mathrm{D}(x, y) \leqslant \mathrm{C} \varphi\left(\limsup _{n \rightarrow \infty} \mathrm{D}\left(x_{n}, y\right)\right)=\mathrm{C} \varphi(0)=0,
$$

which implies from the property $\left(\mathrm{RGM}_{1}\right)$ that $x=y$.

Example 2.7. Let $X=\left\{1-\frac{1}{n}: n \in \mathbb{Z}^{+}\right\} \cup\{1,2\}, \mathbb{Z}^{+}$be the set of positive integers. For any $(x, y) \in X \times X$, define the distances

$$
\begin{aligned}
& D_{g}(x, y)=|x-y|, x, y \in\left\{1-\frac{1}{n}: n \in \mathbb{Z}^{+}\right\}, \\
& D_{g}(x, 1)=D_{g}(1, x)=|x-1|, x \in\left\{1-\frac{1}{n}: n \in \mathbb{Z}^{+}\right\}, \\
& D_{g}(x, 2)=D_{g}(2, x)=|x-2|, x \in\left\{1-\frac{1}{n}: n \in \mathbb{Z}^{+}\right\}, \\
& D_{g}(1,1)=0, D_{g}(2,2)=1, D_{g}(1,2)=D_{g}(2,1)=5 .
\end{aligned}
$$

It is clear that for a function

$$
\varphi(t)= \begin{cases}(t+1)^{2}-1, & t \in[0,1) \\ 3 t, & t \in[1,+\infty)\end{cases}
$$


there exist positive real numbers $C \geqslant \frac{5}{3}$ and an associated number $M=3$ of $\varphi$ such that $\left(\mathrm{RGM}_{3}\right)$ holds for any $x, y \in X$. In fact, it can be observed that the sequence $\left\{1-\frac{1}{n}\right\}_{\mathfrak{n} \in \mathbb{Z}^{+}} \subset X$ converges to 1 , which implies $\left\{1-\frac{1}{n}\right\}_{\mathfrak{n} \in \mathbb{Z}^{+}}$belongs to $\mathscr{C}\left(D_{g}, X, 1,0\right)$. When $x=1$, the inequality $D_{g}(x, y) \leqslant C \varphi\left(\limsup _{n \rightarrow \infty} D_{g}\left(x_{n}, y\right)\right)$ holds for any $y \in X$. In addition, the conditions $\left(R_{G M}\right)$ and $\left(R_{G M}\right)$ are obviously satisfied, thus $\left(X, D_{g}, C, \varphi\right)$ is a re-defined generalized metric space.

Definition 2.8. Let $\left(X, D_{g}, C, \varphi\right)$ be a re-defined generalized metric space, $\left\{x_{n}\right\}_{\mathfrak{n} \in \mathbb{N}}$ be a sequence in $X$. We call $\left\{x_{n}\right\}_{n \in \mathbb{N}}$ a $D_{g}$-Cauchy sequence, if

$$
\lim _{m, n \rightarrow \infty} D_{g}\left(x_{m}, x_{n}\right)=0 .
$$

Definition 2.9. Let $\left(X, D_{g}, C, \varphi\right)$ be a re-defined generalized metric space, $\left\{x_{n}\right\}_{n} \in \mathbb{N}$ be a sequence in $X$. It is said to be $D_{g}$-complete, if every Cauchy sequence in $X$ is convergent to some element in $X$.

\subsection{The relationships between other spaces and the re-defined generalized metric space and example}

To be better able to show the relationships between the standard metric space and the re-defined generalized metric space, let us review the standard metric space, though readers are very familiar with it.

Let $X$ be a nonempty set, the function $d: X \times X \rightarrow[0,+\infty)$ satisfies

$\left(d_{1}\right) d(x, y)=0$ if and only if $x=y$;

$\left(d_{2}\right) d(x, y)=d(y, x)$ for all $x, y \in X$;

$\left(d_{3}\right) d(x, z) \leqslant d(x, y)+d(y, z)$ for all $x, y, z \in X$.

Then $d$ is called a metric of $X$. The pair $(X, d)$ is called a metric space.

By relaxing the triangle inequality in the definition of standard metric, Czerwik [7] introduced the notion of b-metric space, we review it as follows.

Definition 2.10. Let $X$ be a nonempty set, $K \geqslant 1$ be a real number, and the function $\bar{D}: X \times X \rightarrow[0,+\infty)$ satisfies:

$\left(\mathrm{BM}_{1}\right) \overline{\mathrm{D}}(x, y)=0$ if and only if $x=y$;

$\left(\mathrm{BM}_{2}\right) \overline{\mathrm{D}}(x, y)=\mathrm{D}(y, x)$ for all $x, y \in X$;

$\left(\mathrm{BM}_{3}\right) \overline{\mathrm{D}}(x, z) \leqslant K[\overline{\mathrm{D}}(x, y)+\overline{\mathrm{D}}(y, z)]$ for all $x, y, z \in X$.

Then $\bar{D}$ is called a b-metric. $(X, \bar{D})$ is called a metric-type space or a b-metric space.

By weakening the equivalent condition $d(x, y)=0$ in the definition of standard metric, Hitzler and Seda [10] introduced the notion of dislocated metric which is reviewed as follows.

Definition 2.11. Let $X$ be a nonempty set, the function $D_{d}: X \times X \rightarrow[0,+\infty)$ satisfies:

$\left(D_{1}\right)$ If $D_{d}(x, y)=0$, then $x=y$ for every $(x, y) \in X \times X$;

$\left(\mathrm{DM}_{2}\right) \mathrm{D}_{\mathrm{d}}(\mathrm{x}, \mathrm{y})=\mathrm{D}(\mathrm{y}, \mathrm{x})$ for all $x, y \in X$;

$\left(\mathrm{DM}_{3}\right) \mathrm{D}_{\mathrm{d}}(x, z) \leqslant \mathrm{D}(x, y)+\mathrm{D}(y, z)$ for all $x, y, z \in X$.

Then $D_{d}$ is called a dislocated metric. $\left(X, D_{d}\right)$ is called a dislocated metric space.

In 1950, Nakano introduced the notion of modular spaces as a generalization of metric spaces [17], Chistyakov [3] defined the notion of modular metric spaces in which the metrics were generated by the modular on modular spaces in 2010, the series of definitions are reviewed as follows (see also [12]). 
Definition 2.12. Let $X$ be a linear space over $\mathbb{R}$. A function $\mathfrak{m}: X \rightarrow[0,+\infty)$ is called a modular on $X$, if $\left(\mathfrak{m}_{1}\right) \mathfrak{m}(x)=0$ iff $x=\mathbf{0}$,

$\left(\mathfrak{m}_{2}\right) \mathfrak{m}(-\chi)=\mathfrak{m}(x)$ holds for any $x \in X$,

$\left(\mathfrak{m}_{3}\right) \mathfrak{m}(a x+b y) \leqslant \mathfrak{m}(x)+\mathfrak{m}(y)$ for any $(x, y) \in X \times X$,

where $a, b \in \mathbb{R}$ and $a+b=1$.

Definition 2.13. Let $\mathfrak{m}: X \rightarrow[0,+\infty)$ be a modular on $X$, we say the set

$$
X_{\mathfrak{m}}=\left\{x \in X: \lim _{\kappa \rightarrow \infty} \mathfrak{m}(\kappa x)=0\right\},
$$

is a modular space.

A sequence $\left\{x_{\mathfrak{n}}\right\}_{\mathfrak{n} \in N} \subset X_{\mathfrak{m}}$ is said to be $\mathfrak{m}$-convergent to $x \in X_{\mathfrak{m}}$, if

$$
\lim _{n \rightarrow \infty} \mathfrak{m}\left(x_{n} x\right)=0 \text {. }
$$

There exists a metric $D_{\mathfrak{m}}$ induced by the modular $\mathfrak{m}$ of $X$ and defined by

$$
D_{\mathfrak{m}}: X \times X \rightarrow[0,+\infty), D_{\mathfrak{m}}(x, y)=\mathfrak{m}(x-y)
$$

for any $(x, y) \in X \times X .\left(X_{\mathfrak{m}}, D_{\mathfrak{m}}\right)$ is called a modular metric space.

Definition 2.14. The modular $m$ is said to has the Fatou property, if for any $y \in X_{\mathfrak{m}}$, we have

$$
\lim _{n \rightarrow \infty} \mathfrak{m}(x y) \leqslant \liminf _{n \rightarrow \infty} \mathfrak{m}\left(x_{n}-y\right),
$$

where $\left\{x_{\mathfrak{n}}\right\}_{\mathfrak{n} \in \mathbb{N}} \subset X_{\mathfrak{m}}$ is $\mathfrak{m}$-convergent to $x \in X_{\mathfrak{m}}$.

The convergent and complete concepts in the b-metric spaces, the dislocated metric spaces, and the modular metric space are similar to that of standard metric spaces, so we do not repeat them here.

Because all of the standard metrics $d$, the b-metrics $\bar{D}$, the dislocated metrics $D_{d}$, and the modular metrics $D_{\mathfrak{m}}$, where $\mathfrak{m}$ is with Fatou property, are generalized metric (Ref. [12]), therefore as a corollary of Proposition 2.4, the following statement is given.

Corollary 2.15. All of the standard metrics, the dislocated metrics, and the metrics $\mathrm{D}_{\mathfrak{m}}$, which $\mathfrak{m}$ are with Fatou property, are re-defined generalized metric.

\section{The fixed point theorem for the contraction mapping in the re-defined generalized metric space}

In this section, first of all, let us review the definition of contraction mapping on the standard metric space, and then we apply contraction mappings which, of course, is different from that on the standard metric space due to the different metrics to the re-defined generalized metric space. Secondly, we present a fixed point theorem on the re-defined generalized metric space and state that it applies to some special re-defined generalized metric spaces mentioned in previous section. In addition, we have also provided an example to support the conclusions of fixed point.

Let $(X, d)$ be a metric space and $f: X \rightarrow X$ a mapping. If $f$ satisfies the condition

$$
d(f(x), f(y)) \leqslant k d(x, y),
$$

for all $x, y \in X$ and for some $0 \leqslant k<1$, then $f$ is called a contraction.

Definition 3.1. Let $f: X \rightarrow X$ be a self-mapping of a re-defined generalized metric space $X, O(x, f)=$ $\left\{x, f(x), f^{2}(x), \ldots\right\}$ is called the orbit of $x$.

Next let us state the fixed point theorem applied in re-defined generalized metric spaces. 
Theorem 3.2. Let $\left(\mathrm{X}, \mathrm{D}_{\mathrm{g}}, \mathrm{C}, \varphi\right)$ be a $\mathrm{D}_{\mathrm{g}}$-complete re-defined generalized metric space, and $\mathrm{f}: \mathrm{X} \rightarrow \mathrm{X}$ be a mapping satisfying the conditions:

$$
D_{g}(f(x), f(y)) \leqslant k D_{g}(x, y), \quad 0 \leqslant k<1,
$$

and

$$
\sup _{n} D_{g}\left(f^{n}(x), x\right)<\infty, \quad n \in \mathbb{N},
$$

for any $x, y \in X$. Then $\left\{\mathrm{f}^{\mathfrak{n}}\left(\mathrm{x}_{0}\right)\right\}_{\mathfrak{n} \in \mathbb{N}}$ is a convergent sequence for each $\mathrm{x}_{0} \in \mathrm{X}$. In addition, if $\left\{\mathrm{f}^{\mathrm{n}}\left(\mathrm{x}_{0}\right)\right\}_{\mathfrak{n} \in \mathbb{N}}$ is convergent to $\zeta$ uniquely, then $f$ has a fixed point $\zeta$. Moreover, if there exists another fixed point $\zeta^{*}$ such that $\sup _{\mathrm{n}} \mathrm{D}_{\mathrm{g}}\left(\mathrm{f}^{\mathrm{n}}\left(\mathrm{x}_{0}\right), \zeta\right)<\infty, \mathrm{n} \in \mathbb{N}$, then $\zeta^{*}=\zeta$.

Proof. Let $\mathrm{O}(\mathrm{x}, \mathrm{f})=\left\{x, \mathrm{f}(\mathrm{x}), \mathrm{f}^{2}(\mathrm{x}), \ldots\right\}$ be the orbit of $x$. Denote $x_{n}=\mathrm{f}^{\mathrm{n}}(\mathrm{x}), \mathrm{x}_{0}=x$. We will show the sequence $\left\{x_{n}\right\}_{n \in \mathbb{N}}$ is a convergent sequence. For any $x_{n+m}, x_{n} \in O(x, f)$ and $m, n \in \mathbb{N}$, we have

$$
\begin{aligned}
D_{g}\left(x_{n+m}, x_{n}\right) & =D_{g}\left(f \circ f^{m}\left(x_{n-1}\right), f\left(x_{n-1}\right)\right) \\
\leqslant & k\left(D_{g}\left(f^{m}\left(x_{n-1}\right), x_{n-1}\right)\right) \\
= & k\left(D_{g}\left(f \circ f^{m}\left(x_{n-2}\right), f\left(x_{n-2}\right)\right)\right) \\
\leqslant & k^{2}\left(D_{g}\left(f^{m}\left(x_{n-2}\right), x_{n-2}\right)\right) \\
& \vdots \\
\leqslant & k^{n}\left(D_{g}\left(f^{m}\left(x_{0}\right), x_{0}\right)\right),
\end{aligned}
$$

hence, $D_{g}\left(x_{n+m}, x_{n}\right) \leqslant k^{n} \sup D_{g}\left(f^{m}\left(x_{0}\right), x_{0}\right)$, because $\sup D_{g}\left(f^{m}\left(x_{0}\right), x_{0}\right)<\infty$ and $k \in[0,1)$, then it is obvious that $D_{g}\left(x_{n+m}, x_{n}\right)^{m} \rightarrow 0$ as $m, n \rightarrow \infty$, thus the sequence $\left\{x_{n}\right\}_{n \in \mathbb{N}}$ is Cauchy. Since $X$ is $D_{g}$-complete, then the limit $\zeta$ of the sequence $\left\{x_{n}\right\}_{n} \in \mathbb{N}$ belongs to $X$.

Next, let us prove that $\zeta$ is a fixed point of $f$. Because $f$ is a contractive mapping for any $n \in \mathbb{N}$, therefore

$$
D_{g}\left(f(\zeta), f^{n+1}\left(x_{0}\right)\right) \leqslant k D_{g}\left(\zeta, f^{n}\left(x_{0}\right)\right),
$$

it follows from $f^{n}\left(x_{0}\right) \stackrel{D_{g}}{\longrightarrow} \zeta$ that $f^{n}\left(x_{0}\right)$ converges to $f(\zeta)$. By the hypothesis of uniqueness of limit, we have $f(\zeta)=\zeta$.

If there exists another fixed point $\zeta^{*}$ such that $\sup \mathrm{D}_{\mathrm{g}}\left(\mathrm{f}^{\mathfrak{n}}\left(x_{0}\right), \zeta^{*}\right)<\infty$, then

$$
D_{g}\left(\zeta, \zeta^{*}\right)=D_{g}\left(f(\zeta), f\left(\zeta^{*}\right)\right) \leqslant k D_{g}\left(\zeta, \zeta^{*}\right) \leqslant k C \varphi\left(\lim _{n \rightarrow \infty} \sup _{n} D_{g}\left(f^{n}\left(x_{0}\right), \zeta^{*}\right)\right)
$$

Because $\varphi(t)$ is continuous when $t \geqslant 0$ and $\sup D_{g}\left(f^{n}\left(x_{0}\right), \zeta^{*}\right)<\infty$, therefore

$$
k C \varphi\left(\lim _{n \rightarrow \infty} \sup _{n} D_{g}\left(f^{n}\left(x_{0}\right), \zeta^{*}\right)\right)<\infty .
$$

Thus $D_{g}\left(\zeta, \zeta^{*}\right)=D_{g}\left(f(\zeta), f\left(\zeta^{*}\right)\right) \leqslant k D_{g}\left(\zeta, \zeta^{*}\right)<\infty$ which implies $D_{g}\left(\zeta, \zeta^{*}\right)=0$. Using the property $\left(\mathrm{RGM}_{1}\right)$, we obtain $\zeta^{*}=\zeta$.

Example 3.3. Let us review Example 2.7. For $X=\left\{1-\frac{1}{n}: n \in \mathbb{Z}^{+}\right\} \cup\{1,2\}$, the metrics between elements are listed as follows:

$$
\begin{aligned}
& D_{g}(x, y)=|x-y|, x, y \in\left\{1-\frac{1}{n}: n \in \mathbb{Z}^{+}\right\}, \\
& D_{g}(x, 1)=D_{g}(1, x)=|x-1|, x \in\left\{1-\frac{1}{n}: n \in \mathbb{Z}^{+}\right\}, \\
& D_{g}(x, 2)=D_{g}(2, x)=|x-2|, x \in\left\{1-\frac{1}{n}: n \in \mathbb{Z}^{+}\right\}, \\
& D_{g}(1,1)=0, D_{g}(2,2)=1, D_{g}(1,2)=D_{g}(2,1)=5 .
\end{aligned}
$$


It is easy to see that there exist two Cauchy sequences $\left\{1-\frac{1}{n}\right\}_{\mathfrak{n} \in \mathbb{Z}^{+}}$and $\left\{x_{\mathfrak{n}}=1\right\}_{\mathfrak{n} \in \mathbb{Z}^{+}}$in $X$, while others are the sub-sequence of $\left\{1-\frac{1}{n}\right\}_{n \in \mathbb{Z}^{+}}$, all the Cauchy sequences converge to $1 \in X$, thus $\left(X, D_{g}, C, \varphi\right)$ be a $\mathrm{D}_{\mathrm{g}}$-complete re-defined generalized metric space. Define the mapping

$$
f\left(1-\frac{1}{n}\right)=1-\frac{1}{2(n+1)}, n \in \mathbb{Z}^{+}, \quad f(1)=1, \quad f(2)=1 .
$$

By a simple calculation, one can show

$$
\begin{aligned}
& D_{g}\left(f\left(1-\frac{1}{m}\right), f\left(1-\frac{1}{n}\right)\right)=\frac{|m-n|}{2(m+1)(n+1)}, \quad m, n \in \mathbb{Z}^{+}, \\
& D_{g}(f(1), f(1))=D_{g}(f(2), f(2))=D_{g}(f(1), f(2))=0, \\
& D_{g}\left(f(1), f\left(1-\frac{1}{n}\right)\right)=D_{g}\left(f(2), f\left(1-\frac{1}{n}\right)\right)=\frac{1}{2(n+1)}, \quad n \in \mathbb{Z}^{+} .
\end{aligned}
$$

It can be verified that when we choose $\frac{1}{2} \leqslant k<1$, the following inequality will be satisfied for any $x, y \in X$.

$$
D_{g}(f(x), f(y)) \leqslant k D_{g}(x, y) .
$$

If we choose $x=1-\frac{1}{m}$ for every fixed $m \in \mathbb{N}$, then when $n \rightarrow \infty$,

$$
\mathrm{f}^{\mathrm{n}}\left(1-\frac{1}{\mathrm{~m}}\right)=1-\frac{1}{2^{\mathrm{n}}(\mathrm{m}+1)+2 \mathrm{n}} \stackrel{\mathrm{D}_{\mathrm{g}}}{\longrightarrow} 1 .
$$

In addition, it is clear that

$$
D_{g}\left(f^{n}(x), x\right)=\frac{\left|2^{n}(m+1)+2 n-m\right|}{\left(2^{n}(m+1)+2 n\right) m}<\infty, \quad n \in \mathbb{Z}^{+},
$$

for fixed $m \in \mathbb{Z}^{+}$, thus $\sup D_{g}\left(f^{n}(x), x\right)<\infty, n \in \mathbb{Z}^{+}$.

Similarly, one can verify that whether $x=1$ or $x=2$, the facts

$$
f^{n}(x) \stackrel{D_{g}}{\longrightarrow} 1 \text { and } \sup _{n} D_{g}\left(f^{n}(x), x\right)<\infty
$$

hold. All conditions of Theorem 3.2 are met in term of this example. As we have seen, there is really a unique fixed point $1 \in X$.

Lemma 3.4. Let $\left(\mathrm{X}, \mathrm{D}_{\mathrm{g}}, \mathrm{C}, \varphi\right)$ be a $\mathrm{D}_{\mathrm{g}}$-complete re-defined generalized metric space, and $\mathrm{f}: \mathrm{X} \rightarrow \mathrm{X}$ be a mapping satisfying the condition:

$$
D_{g}(f(x), f(y)) \leqslant k D_{g}(x, y), \quad 0 \leqslant k<1
$$

If

$$
\sup _{n} D_{g}\left(f^{n}(x), x\right)<\infty, \quad n \in \mathbb{N}
$$

holds for any $x, y \in X$, then

$$
\delta\left(D_{g}, f, x\right)=\sup \left\{D_{g}\left(f^{i}(x), f^{j}(x)\right), i, j \in \mathbb{N}\right\}<\infty .
$$

In this case, it has been shown that $\left\{\mathrm{f}^{\mathfrak{n}}\left(\mathrm{x}_{0}\right)\right\}_{\mathfrak{n} \in \mathbb{N}}$ is a convergent sequence for each $\mathrm{x}_{0} \in \mathrm{X}$ and if $\left\{\mathrm{f}^{\mathrm{n}}\left(\mathrm{x}_{0}\right)\right\}_{\mathfrak{n} \in \mathbb{N}}$ is convergent to $\zeta$ uniquely, $f$ has a fixed point $\zeta$. Moreover, if there exists another fixed point $\zeta^{*}$ such that $\sup _{n} D_{g}\left(f^{n}\left(x_{0}\right), \zeta^{*}\right)<\infty, n \in \mathbb{N}$, then $D_{g}\left(\zeta, \zeta^{*}\right)<\infty$. 
Proof. There exists $\mathrm{O}(x, f)$ for every $x \in X$ such that $x \in O(x, f)$, the condition $\sup _{m} D\left(f^{m}(x), x\right)<\infty$ implies

$$
\sup _{m} D\left(f^{m}\left(x_{n}\right), x_{n}\right)=\sup _{m} D\left(f^{n+m}(x), f^{n}(x)\right)<\infty,
$$

for every fixed $n \in \mathbb{N}$ and all $m \in \mathbb{N}$, hence

$$
\delta\left(D_{g}, f, x\right)=\sup \left\{D_{g}\left(f^{i}(x), f^{j}(x)\right), i, j \in \mathbb{N}\right\}<\infty .
$$

If there exists another fixed point $\zeta^{*}$ such that $\sup _{n} D_{g}\left(f^{n}\left(x_{0}\right), \zeta^{*}\right)<\infty$, then by $\left(R_{G M}\right)$,

$$
D_{g}\left(\zeta, \zeta^{*}\right) \leqslant C \varphi\left(\lim _{n \rightarrow \infty} \sup _{n} D_{g}\left(f^{n}\left(x_{0}\right), \zeta^{*}\right)\right)
$$

Because $\varphi(t)$ is continuous when $t \geqslant 0$ and $\sup _{n} D_{g}\left(f^{n}\left(x_{0}\right), \zeta^{*}\right)<\infty$, therefore

$$
\mathrm{C} \varphi\left(\lim _{n \rightarrow \infty} \sup _{n} D_{g}\left(f^{n}\left(x_{0}\right), \zeta^{*}\right)\right)<\infty
$$

Lemma 3.5 ([12]). Let (X, D) be a generalized metric space and $\left\{x_{n}\right\}$ be a sequence in $X,(x, y) \in X \times X$. If $\left\{x_{n}\right\}$ is $\mathrm{D}$-convergent in $\mathrm{X}$, then the limit is unique.

The subsequent corollary (Jleli and Samet, ref. Theorem 3.3 in [12]) is an immediate consequence of of Proposition 2.4, Theorem 3.2, Lemma 3.5, and Lemma 3.4.

Corollary 3.6. Let $(\mathrm{X}, \mathrm{D})$ be a $\mathrm{D}$-complete generalized metric space, and $\mathrm{f}: \mathrm{X} \rightarrow \mathrm{X}$ be a mapping satisfying the conditions:

$$
D(f(x), f(y)) \leqslant k D(x, y), \quad 0 \leqslant k<1,
$$

and

$$
\delta(D, f, x)=\sup \left\{D_{g}\left(f^{i}(x), f^{j}(x)\right), i, j \in \mathbb{N}\right\}<\infty,
$$

for any $x, y \in X$. Then $\mathrm{f}$ has a fixed point $\zeta$ and $\mathrm{f}^{\mathrm{n}}\left(\mathrm{x}_{0}\right)$ converges to $\zeta$ for each $\mathrm{x}_{0} \in \mathrm{X}$. If there exists another fixed point $\zeta^{*}$ such that $\mathrm{D}\left(\zeta, \zeta^{*}\right)<\infty, \mathrm{n} \in \mathbb{N}$, then $\zeta^{*}=\zeta$.

Because all of the standard metrics $d$, the b-metrics $\bar{D}$, the dislocated metrics $D_{d}$, and the modular metrics $D_{\mathfrak{m}}$, where $\mathfrak{m}$ is with Fatou property, are generalized metric ([12]), the subsequent several corollaries can be derived directly from Corollary 2.15 and Corollary 3.6.

Corollary 3.7. Let $(\mathrm{X}, \mathrm{d})$ be a complete $\mathrm{b}$-metric space, and $\mathrm{f}: \mathrm{X} \rightarrow \mathrm{X}$ be a mapping satisfying the conditions:

$$
d(f(x), f(y)) \leqslant k d(x, y), \quad 0 \leqslant k<1,
$$

and

$$
\sup _{n} d\left(f^{n}(x), x\right)<\infty, \quad n \in \mathbb{N},
$$

for any $\mathrm{x}, \mathrm{y} \in \mathrm{X}$. Then $\mathrm{f}$ has a fixed point $\zeta$ and $\mathrm{f}^{\mathrm{n}}\left(\mathrm{x}_{0}\right)$ converges to $\zeta$ for each $\mathrm{x}_{0} \in \mathrm{X}$. If there exists another fixed point $\zeta^{*}$ such that $\sup _{\mathrm{n}} \mathrm{d}\left(\mathrm{f}^{\mathrm{n}}\left(\mathrm{x}_{0}\right), \zeta^{*}\right)<\infty, \mathrm{n} \in \mathbb{N}$, then $\zeta^{*}=\zeta$.

Corollary 3.8. Let $(\mathrm{X}, \overline{\mathrm{D}})$ be a complete $\mathrm{b}$-metric space, and $\mathrm{f}: \mathrm{X} \rightarrow \mathrm{X}$ be a mapping satisfying the conditions:

$$
\bar{D}(f(x), f(y)) \leqslant k \bar{D}(x, y), \quad 0 \leqslant k<1,
$$

and

$$
\sup _{n} \bar{D}\left(f^{n}(x), x\right)<\infty, \quad n \in \mathbb{N}
$$

for any $x, y \in X$. Then $\mathrm{f}$ has a fixed point $\zeta$ and $\mathrm{f}^{\mathrm{n}}\left(\mathrm{x}_{0}\right)$ converges to $\zeta$ for each $\mathrm{x}_{0} \in \mathrm{X}$. If there exists another fixed point $\zeta^{*}$ such that $\sup _{n} \bar{D}\left(f^{n}\left(x_{0}\right), \zeta^{*}\right)<\infty, n \in \mathbb{N}$, then $\zeta^{*}=\zeta$. 
Corollary 3.9. Let $\left(\mathrm{X}, \mathrm{D}_{\mathrm{d}}\right)$ be a complete dislocated metric space, and $\mathrm{f}: \mathrm{X} \rightarrow \mathrm{X}$ be a mapping satisfying the conditions:

$$
D_{d}(f(x), f(y)) \leqslant k D_{d}(x, y), \quad 0 \leqslant k<1,
$$

and

$$
\sup _{n} D_{d}\left(f^{n}(x), x\right)<\infty, \quad n \in \mathbb{N},
$$

for any $x, y \in X$. Then $\mathrm{f}$ has a fixed point $\zeta$ and $\mathrm{f}^{\mathrm{n}}\left(\mathrm{x}_{0}\right)$ converges to $\zeta$ for each $\mathrm{x}_{0} \in \mathrm{X}$. If there exists another fixed point $\zeta^{*}$ such that $\sup _{\mathrm{n}} \mathrm{D}_{\mathrm{d}}\left(\mathrm{f}^{\mathrm{n}}\left(\mathrm{x}_{0}\right), \zeta^{*}\right)<\infty, \mathrm{n} \in \mathbb{N}$, then $\zeta^{*}=\zeta$.

Corollary 3.10. Let $\left(\mathrm{X}, \mathrm{D}_{\mathfrak{m}}\right)$ be a complete modular space with Fatou property, and $\mathrm{f}: \mathrm{X} \rightarrow \mathrm{X}$ be a mapping satisfying the conditions:

$$
D_{\mathfrak{m}}(f(x), f(y)) \leqslant k D_{\mathfrak{m}}(x, y), \quad 0 \leqslant k<1,
$$

and

$$
\sup _{n} D_{\mathfrak{m}}\left(f^{\mathfrak{n}}(x), x\right)<\infty, \quad n \in \mathbb{N},
$$

for any $x, y \in X$. Then $\mathrm{f}$ has a fixed point $\zeta$ and $\mathrm{f}^{\mathrm{n}}\left(\mathrm{x}_{0}\right)$ converges to $\zeta$ for each $\mathrm{x}_{0} \in \mathrm{X}$. If there exists another fixed point $\zeta^{*}$ such that $\sup _{\mathfrak{n}} \mathrm{D}_{\mathfrak{m}}\left(\mathrm{f}^{\mathrm{n}}\left(\mathrm{x}_{0}\right), \zeta^{*}\right)<\infty, \mathrm{n} \in \mathbb{N}$, then $\zeta^{*}=\zeta$.

\section{The fixed point theorem related to Ćirić-Maiti-Pal orbit mapping of contractive type in re-defined generalized metric spaces}

Wong [26] and Guseman [9] have studied the mappings which are contractive over an orbit. Here we apply such mappings into the re-defined generalized metric spaces.

Definition 4.1. Let $f: X \rightarrow X$ be a self-mapping of a re-defined generalized metric space $X, O(x, f)$ is the orbit of $x$. The closure of $O(x, f)$ will be denoted by $\bar{O}(x, f)$. The space $X$ is said to be $f$-orbitally complete, if every Cauchy sequence contained in $O(x, f)$ converges in $X$, for all $x \in X$.

In 1974, Cirić [4] defined quasi-contractive mappings and stated some fixed point results in which it has shown that the condition of quasi-contractivity implies all conclusions of Banach's contraction principle. The definition was extended by Pal and Maiti [20] subsequently and the following mapping of contractive type was presented.

Let $X$ denote a complete metric space with metric $d$, and $f$ a function mapping $X$ into itself. There exists a constant $h, 0<h<1$, such that for each $x, y \in X$, the following is true:

$$
d(f(x), f(y)) \leqslant h \max \left\{d(x, y), d(x, f(y)), d(y, f(y)), \frac{1}{2}[d(x, f(y))+d(y, f(x))]\right\},
$$

here we extend it to the Ćirić-Maiti-Pal orbit mapping of contractive type which is shown as follows.

Definition 4.2. Let $f: X \rightarrow X$ be a map on a re-defined generalized metric space $\left(X, D_{g}, C, \varphi\right)$ and $X$ is f-orbitally complete. If there exists an element $x \in X$ such that for any elements $u, v \in \bar{O}(x, f), u$ and $v$ do not belong to $\bar{O}(x, f) \backslash O(x, f)$ together and there exists $0<\alpha<1$, the following is true:

$$
D_{g}(f(u), f(v)) \leqslant \alpha \max \left\{D_{g}(u, v), D_{g}(u, f(u)), D_{g}(v, f(v)), \frac{1}{2}\left[D_{g}(u, f(v))+D_{g}(v, f(u))\right]\right\},
$$

then $f$ is called a Ćirić-Maiti-Pal orbit mapping of contractive type.

Theorem 4.3. Let $\mathrm{f}: \mathrm{X} \rightarrow \mathrm{X}$ be a Ćirić-Maiti-Pal orbit mapping of contractive type on a re-defined generalized metric space $(X, D, C, \varphi)$ and $X$ is f-orbitally complete, that is, there exists an element $\mathrm{X} \in \mathrm{X}$ such that for any elements $\mathrm{u}, v \in \overline{\mathrm{O}}(\mathrm{x}, \mathrm{f})$, $\mathrm{u}$ and $v$ do not belong to $\overline{\mathrm{O}}(\mathrm{x}, \mathrm{f}) \backslash \mathrm{O}(\mathrm{x}, \mathrm{f})$ together and there exists $0<\alpha<1$, the following 
inequality holds:

$$
D_{g}(f(u), f(v)) \leqslant \alpha \max \left\{D_{g}(u, v), D_{g}(u, f(u)), D_{g}(v, f(v)), \frac{1}{2}\left[D_{g}(u, f(v))+D_{g}(v, f(u))\right]\right\},
$$

then we have the following results:

If $\sup _{\mathrm{n}} \mathrm{D}\left(\mathrm{f}^{\mathrm{n}}(\mathrm{x}), \mathrm{x}\right)<\infty$ for all $\mathrm{x} \in \mathrm{X}$, then $\left\{\mathrm{f}^{\mathrm{n}}\left(\mathrm{x}_{0}\right)\right\}_{\mathfrak{n} \in \mathbb{N}}$ is convergent to some $\zeta$. Moreover, let $\mathrm{M}$ be an associated number of $\varphi$, if $\left\{\mathrm{f}^{\mathrm{n}}\left(\mathrm{x}_{0}\right)\right\}_{\mathfrak{n} \in \mathbb{N}}$ is convergent to $\zeta$ uniquely and $\mathrm{CM} \alpha<1$, then $\zeta$ is a fixed point of $\mathrm{f}$.

Proof. For $x \in \mathrm{O}(x, \mathrm{f})$, the condition $\sup _{\mathrm{m}} \mathrm{D}\left(\mathrm{f}^{\mathrm{m}}(x), x\right)<\infty$ implies

$$
\sup _{m} D\left(f^{m}\left(x_{n}\right), x_{n}\right)=\sup _{m} D\left(f^{n+m}(x), f^{n}(x)\right)<\infty,
$$

for every fixed $n \in \mathbb{N}$ and all $m \in \mathbb{N}$. Hence

$$
\sup _{m_{1}, m_{2}} D\left(f^{m_{1}}\left(x_{n}\right), f^{m_{2}}\left(x_{n}\right)\right)=\sup _{m_{1}, m_{2}} D\left(f^{n+m_{1}}(x), f^{n+m_{2}}(x)\right)<\infty,
$$

for all $n, m_{1}, m_{2} \in \mathbb{N}$. We denote $\sup _{m_{1}, m_{2}} D\left(f^{m_{1}}\left(x_{n}\right), f^{m_{2}}\left(x_{n}\right)\right)$ by $\mathfrak{B}_{n}(x, f)$ for every fixed $n \in \mathbb{N}$ and all $m_{1}, m_{2} \in \mathbb{N}$. Because $f$ is a Ćirić-Maiti-Pal orbit mapping of contractive type, then for every fixed $n \in \mathbb{N}$ and all $\mathrm{m}_{1}, \mathrm{~m}_{2} \in \mathbb{N}$, we have

$$
\begin{aligned}
D_{g}\left(f^{m_{1}}\left(x_{n+1}\right), f^{m_{2}}\left(x_{n+1}\right)\right)= & D_{g}\left(f\left(x_{n+m_{1}}\right), f\left(x_{n+m_{2}}\right)\right) \\
\leqslant & \alpha \max \left\{D_{g}\left(x_{n+m_{1}}, x_{n+m_{2}}\right), D_{g}\left(x_{n+m_{1}}, f\left(x_{n+m_{1}}\right)\right), D_{g}\left(x_{n+m_{2}}, f\left(x_{n+m_{2}}\right)\right),\right. \\
& \left.\frac{1}{2}\left[D_{g}\left(x_{n+m_{1}}, f\left(x_{n+m_{2}}\right)\right)+D_{g}\left(x_{n+m_{2}}, f\left(x_{n+m_{1}}\right)\right)\right]\right\} \\
\leqslant & \alpha \max \left\{D_{g}\left(f^{m_{1}}\left(x_{n}\right), f^{m_{2}}\left(x_{n}\right)\right), D_{g}\left(f^{m_{1}}\left(x_{n}\right), f^{m_{1}+1}\left(x_{n}\right)\right), D_{g}\left(f^{m_{2}}\left(x_{n}\right),\right.\right. \\
& \left.\left.f^{m_{2}+1}\left(x_{n}\right)\right), \frac{1}{2}\left[D_{g}\left(f^{m_{1}}\left(x_{n}\right), f^{m_{2}+1}\left(x_{n}\right)\right)+D_{g}\left(f^{m_{2}}\left(x_{n}\right), f^{m_{1}+1}\left(x_{n}\right)\right)\right]\right\} .
\end{aligned}
$$

Note that

$$
\sup _{m_{1}, m_{2}} D_{g}\left(f^{m_{1}}\left(x_{n}\right), f^{m_{2}+1}\left(x_{n}\right)\right)=\sup _{m_{1}, m_{2}} D_{g}\left(f^{m_{2}}\left(x_{n}\right), f^{m_{1}+1}\left(x_{n}\right)\right)
$$

then

$$
\sup _{m_{1}, m_{2}} D_{g}\left(f^{m_{1}}\left(x_{n+1}\right), f^{m_{2}}\left(x_{n+1}\right)\right) \leqslant \alpha \sup _{m_{1}, m_{2}} D_{g}\left(f^{m_{2}}\left(x_{n}\right), f^{m_{1}}\left(x_{n}\right)\right)
$$

that is,

$$
\mathfrak{B}_{\mathfrak{n}+1}(x, f) \leqslant \alpha \mathfrak{B}_{\mathfrak{n}}(\chi, f),
$$

for all $n \in \mathbb{N}$. Subsequently,

$$
D_{g}\left(f^{n}(x), f^{n+m}(x)\right) \leqslant \mathfrak{B}_{n}(x, f) \leqslant \alpha^{n} \mathfrak{B}_{0}(x, f) .
$$

Because $\alpha \in(0,1)$ and $\mathfrak{B}_{0}(x, f)$ is bounded, therefore $D_{g}\left(f^{n}(x), f^{n+m}(x)\right) \rightarrow 0$ when $n \rightarrow \infty$, which implies that $\left\{f^{\mathfrak{n}}(x)\right\}_{\mathfrak{n} \in \mathbb{N}}$ is Cauchy. On the other hand, since $X$ be f-orbitally complete, then the limit $\zeta$ of the sequence $\left\{\mathrm{f}^{\mathfrak{n}}(\mathrm{x})\right\}_{\mathfrak{n} \in \mathbb{N}}$ belongs to $X$.

Next, let us prove that $\zeta$ is a fixed point of $f$.

If $D_{g}(\zeta, f(\zeta))=0$, then it is obvious from $\left(R_{G M}\right)$ that $f(\zeta)=\zeta$. 
If $D_{g}(\zeta, f(\zeta))>0$, using $\left(R_{G M}\right)$, one can obtain that

$$
D_{g}(\zeta, f(\zeta)) \leqslant C \varphi\left(\limsup _{n \rightarrow \infty} D_{g}\left(f^{n}\left(x_{0}\right), f(\zeta)\right)\right)
$$

Hence

$$
\begin{gathered}
D_{g}\left(f^{n}\left(x_{0}\right), f(\zeta)\right) \leqslant \alpha \max \left\{D_{g}\left(f^{n-1}\left(x_{0}\right), \zeta\right), D_{g}\left(f^{n-1}\left(x_{0}\right), f^{n}\left(x_{0}\right)\right), D_{g}(\zeta, f(\zeta)),\right. \\
\left.\frac{1}{2}\left[D_{g}\left(f^{n-1}\left(x_{0}\right), f(\zeta)\right)+D_{g}\left(\zeta, f^{n}\left(x_{0}\right)\right)\right]\right\},
\end{gathered}
$$

holds for every positive number $n$. Taking upper limits on the both sides of the inequality,

$$
\begin{aligned}
\limsup _{n \rightarrow \infty} D_{g}\left(f^{n}\left(x_{0}\right), f(\zeta)\right) \leqslant & \alpha \max \left\{\limsup _{n \rightarrow \infty} D_{g}\left(f^{n-1}\left(x_{0}\right), \zeta\right), \underset{n \rightarrow \infty}{\limsup D_{g}\left(f^{n-1}\left(x_{0}\right), f^{n}\left(x_{0}\right)\right),}\right. \\
& \left.D_{g}(\zeta, f(\zeta)), \frac{1}{2}\left[\limsup _{n \rightarrow \infty} D_{g}\left(f^{n-1}\left(x_{0}\right), f(\zeta)\right)+\limsup _{n \rightarrow \infty} D_{g}\left(\zeta, f^{n}\left(x_{0}\right)\right)\right]\right\} .
\end{aligned}
$$

Because $\left\{\mathrm{f}^{\mathfrak{n}}\left(\mathrm{x}_{0}\right)\right\}_{\mathfrak{n} \in \mathbb{N}}$ is a Cauchy sequence and converges to $\zeta$, therefore

$$
\limsup _{n \rightarrow \infty} D_{g}\left(f^{n-1}\left(x_{0}\right), f^{n}\left(x_{0}\right)\right)=\limsup _{n \rightarrow \infty} D_{g}\left(\zeta, f^{n}\left(x_{0}\right)\right)=0,
$$

hence

$$
\limsup _{n \rightarrow \infty} D_{g}\left(f^{n}\left(x_{0}\right), f(\zeta)\right) \leqslant \alpha \max \left\{D_{g}(\zeta, f(\zeta)), \frac{1}{2} \limsup _{n \rightarrow \infty} D_{g}\left(f^{n}\left(x_{0}\right), f(\zeta)\right)\right\}
$$

If

$$
\alpha \max \left\{D_{g}(\zeta, f(\zeta)), \frac{1}{2} \limsup _{n \rightarrow \infty} D_{g}\left(f^{n-1}\left(x_{0}\right), f(\zeta)\right)\right\}=\alpha D_{g}(\zeta, f(\zeta))
$$

in combination with inequality (4.2) and $\mathrm{RGM}_{3}$, we obtain that

$$
\begin{aligned}
D_{g}(\zeta, f(\zeta)) & \leqslant C \varphi\left(\limsup _{n \rightarrow \infty} D_{g}\left(f^{n}\left(x_{0}\right), f(\zeta)\right)\right) \\
& \leqslant C \varphi\left(\alpha D_{g}(\zeta, f(\zeta))\right) \\
& \leqslant C M \alpha D_{g}(\zeta, f(\zeta))
\end{aligned}
$$

it follows from $C M \alpha<1$ that $D_{g}(\zeta, f(\zeta))=0$, which contradicts $D_{g}(\zeta, f(\zeta))>0$, so

$$
\begin{aligned}
\limsup _{n \rightarrow \infty} D_{g}\left(f^{n}\left(x_{0}\right), f(\zeta)\right) & \leqslant \alpha \max \left\{D_{g}(\zeta, f(\zeta)), \frac{1}{2} \limsup _{n \rightarrow \infty} D_{g}\left(f^{n}\left(x_{0}\right), f(\zeta)\right)\right\} \\
& =\frac{\alpha}{2} \limsup _{n \rightarrow \infty} D_{g}\left(f^{n}\left(x_{0}\right), f(\zeta)\right),
\end{aligned}
$$

then from $0<\alpha<1$,

$$
\limsup _{n \rightarrow \infty} D_{g}\left(f^{n}\left(x_{0}\right), f(\zeta)\right)=0,
$$

thus, $\left\{f^{\mathfrak{n}}\left(x_{0}\right)\right\}_{\mathfrak{n} \in \mathbb{N}}$ converges to $f(\zeta)$, by the hypothesis of uniqueness of limit, we have $f(\zeta)=\zeta$.

Remark 4.4. It is worth noting that in Theorem 4.3, the re-defined generalized metric space $\left(X, D_{g}, C, \varphi\right)$ is not necessarily complete, but merely f-orbitally complete. The contractive condition (4.1) of Theorem 4.3 only holds at points in the closure of the orbit of $x$ for some point $x \in X$, rather than for all $x, y \in X$.

Example 4.5. Let $X=\left\{1-\frac{1}{p}, 3-\frac{1}{q}, 5-\frac{1}{r}: p, q, r \in \mathbb{Z}^{+}\right\} \cup\{1,3,7\}$, we denote

$$
X_{1}=\left\{1-\frac{1}{p}, 3-\frac{1}{q}, 5-\frac{1}{r}: p, q, r \in \mathbb{Z}^{+}\right\}
$$


and define the distances

$$
\begin{aligned}
& D_{g}(x, y)=|x-y|, x, y \in X_{1} \\
& D_{g}(x, 1)=D_{g}(1, x)=|x-1|, x \in X_{1} \\
& D_{g}(x, 3)=D_{g}(3, x)=|x-3|, x \in X_{1} \\
& D_{g}(x, 7)=D_{g}(7, x)=|x-7|, x \in X_{1} \\
& D_{g}(1,1)=1, D_{g}(3,3)=0, D_{g}(1,3)=D_{g}(3,1)=1 \\
& D_{g}(7,7)=7, D_{g}(3,7)=D_{g}(7,3)=5, D_{g}(1,7)=D_{g}(7,1)=3 .
\end{aligned}
$$

We can verify that $\left(X, D_{g}, C, \varphi\right)$ is a re-defined generalized metric space when taking $C=2$,

$$
\varphi(t)= \begin{cases}e^{t}-1, & t \in[0,1) \\ (e-1) t, & t \in[1,+\infty)\end{cases}
$$

and $M=e-1$. In addition, the conditions $\left(R G M_{1}\right)$ and $\left(R G M_{2}\right)$ are obviously satisfied. However, it is demonstrated that $\left(X, D_{g}, C, \varphi\right)$ is not a $D_{g}$-complete re-defined generalized metric space since the fact that the Cauchy sequence $\left\{5-\frac{1}{n}\right\}_{\mathfrak{n} \in \mathbb{Z}^{+}}$converges to $5 \notin X$.

Define the mapping

$$
\begin{aligned}
& f(1)=1, \quad f(3)=3, \quad f(7)=3, \quad f\left(1-\frac{1}{p}\right)=1-\frac{1}{40(p+1)}, \\
& f\left(3-\frac{1}{q}\right)=3-\frac{1}{40(q+1)}, \quad f\left(5-\frac{1}{r}\right)=3-\frac{1}{40(r+1)},
\end{aligned}
$$

for any $p, q, r \in \mathbb{Z}^{+}$.

By a simple calculation, one can show that for any $m, n \in \mathbb{Z}^{+}$,

$$
\begin{aligned}
& D_{g}(f(1), \quad f(1))=1, \quad D_{g}(f(3), f(3))=0, \quad D_{g}(f(1), \quad f(3))=1, \\
& D_{g}(f(7), \quad f(7))=0, \quad D_{g}(f(7), \quad f(3))=3, \quad D_{g}(f(7), \quad f(1))=1, \\
& D_{g}\left(f(1), \quad f\left(1-\frac{1}{n}\right)\right)=D_{g}\left(f(3), \quad f\left(3-\frac{1}{n}\right)\right)=D_{g}\left(f(7), \quad f\left(3-\frac{1}{n}\right)\right)=\frac{1}{40(n+1)}, \\
& D_{g}\left(f(1), \quad f\left(3-\frac{1}{n}\right)\right)=D_{g}\left(f(3), \quad f\left(1-\frac{1}{n}\right)\right)=D_{g}\left(f(7), \quad f\left(1-\frac{1}{n}\right)\right)=2-\frac{1}{40(n+1)}, \\
& D_{g}\left(f(1), \quad f\left(5-\frac{1}{n}\right)\right)=2-\frac{1}{40(n+1)}, \\
& D_{g}\left(f(3), \quad f\left(5-\frac{1}{n}\right)\right)=D_{g}\left(f(7), \quad f\left(5-\frac{1}{n}\right)\right)=\frac{1}{40(n+1)}, \\
& D_{g}\left(f\left(l_{1}-\frac{1}{m}\right), \quad f\left(l_{2}-\frac{1}{n}\right)\right)=\frac{|m-n|}{40(m+1)(n+1)}, \quad l_{1}=l_{2} \in\{1,3,5\}, \\
& D_{g}\left(f\left(l_{1}-\frac{1}{m}\right), \quad f\left(l_{2}-\frac{1}{n}\right)\right)=\left|l_{1}-l_{2}\right|-\frac{|m-n|}{40(m+1)(n+1)}, \quad l_{1} \neq l_{2}, \quad l_{1}, l_{2} \in\{1,3,5\},
\end{aligned}
$$

which implies $f$ is not a mapping of contractive type. In fact, one can observe that

$$
D_{g}\left(f(1), f\left(3-\frac{1}{n}\right)\right)=2-\frac{1}{40(n+1)}>D_{g}\left(1,3-\frac{1}{n}\right)=2-\frac{1}{n} .
$$

But we can verify subsequently that $f$ is a Ćirić-Maiti-Pal orbit mapping of contractive type. Let us consider the orbit

$$
O\left(1-\frac{1}{m}, f\right)=\left\{1-\frac{1}{m}, f\left(1-\frac{1}{m}\right), f^{2}\left(1-\frac{1}{m}\right), \ldots, f^{n}\left(1-\frac{1}{m}\right), \ldots\right\},
$$

for every fixed $m \in \mathbb{Z}^{+}$, where

$$
f^{n}\left(1-\frac{1}{m}\right)=1-\frac{1}{40^{n}(m+1)+40 n} .
$$


In term of $\varphi, C$ and $M$ mentioned above in this example, we can choose $\alpha, 0<\alpha<1$ such that $C M \alpha<1$, when $u, v \in \bar{O}\left(1-\frac{1}{m}, f\right)$ and $u$ and $v$ do not belong to $\bar{O}\left(1-\frac{1}{m}, f\right) \backslash O\left(1-\frac{1}{m}, f\right)$ at the same time, the following inequality is true:

$$
D_{g}(f(u), f(v)) \leqslant \alpha \max \left\{D_{g}(u, v), D_{g}(u, f(u)), D_{g}(v, f(v)), \frac{1}{2}\left[D_{g}(u, f(v))+D_{g}(v, f(u))\right]\right\} .
$$

So, it suffices to show that the orbit $\mathrm{O}\left(1-\frac{1}{\mathrm{~m}}, \mathrm{f}\right)$ satisfies the condition $\sup _{\mathrm{n}} \mathrm{D}\left(\mathrm{f}^{\mathrm{n}}\left(1-\frac{1}{\mathrm{~m}}\right), 1-\frac{1}{\mathrm{~m}}\right)<\infty$. In addition, one can see that $f^{n}\left(1-\frac{1}{m}\right) \stackrel{D_{g}}{\longrightarrow} 1$ which implies the element 1 is a fixed point of $f$ from Theorem 4.3. Similarly, if we consider another orbit $\mathrm{O}\left(3-\frac{1}{\mathrm{~m}}, \mathrm{f}\right)$ for every fixed $\mathrm{m} \in \mathbb{Z}^{+}$, then one can derive that the element $3 \in X$ is a fixed point of $f$. Moreover, the orbit $O(7, f)=\left\{7,3=f(7), 3=f^{2}(7), \ldots\right\}$ satisfies the conditions of Theorem 4.3, it can also be demonstrated that the element 3 is a fixed point of $f$. Our results are verified and explained well by this example.

Remark 4.6. Under the conditions of Theorem 4.3, the fixed point of Ćirić-Maiti-Pal orbit mapping of contractive type is not necessarily unique, which can be verified from Example 4.5. This is because, as remarked in Remark 4.4, the contractive conditions (4.1) of Theorem 4.3 only hold at points in the closure of the orbit of $x$ for some point $x \in X$, rather than for all $x, y \in X$.

If the contractive condition (4.1) holds for all $u, v \in X$, then the following theorem can be stated.

Theorem 4.7. Let $\mathrm{f}$ be a mapping of contractive type satisfying the contraction condition

$$
D_{g}(f(u), f(v)) \leqslant \alpha \max \left\{D_{g}(u, v), D_{g}(u, f(u)), D_{g}(v, f(v)), \frac{1}{2}\left[D_{g}(u, f(v))+D_{g}(v, f(u))\right]\right\},
$$

for all $\mathrm{u}, v \in \mathrm{X}$ in the setting of a re-generalized metric space $\left(\mathrm{X}, \mathrm{D}_{\mathrm{g}}, \mathrm{C}, \varphi\right)$ and $\mathrm{X}$ is $\mathrm{D}_{\mathrm{g}}$-complete, $\mathrm{M}$ is an associated number of $\varphi, O(x, f)$ is the orbit of $x$, and $x_{n}=f^{n}(x) \in O(x, f)$, then we have the following results:

If $\sup _{\mathrm{n}} \mathrm{D}\left(\mathrm{f}^{\mathrm{n}}(\mathrm{x}), \mathrm{x}\right)<\infty$ for all $\mathrm{x} \in \mathrm{X}$, then $\left\{\mathrm{f}^{\mathrm{n}}\left(\mathrm{x}_{0}\right)\right\}_{\mathfrak{n} \in \mathbb{N}}$ is convergent to some $\zeta$. In addition, if $\left\{\mathrm{f}^{\mathrm{n}}\left(\mathrm{x}_{0}\right)\right\}_{\mathfrak{n} \in \mathbb{N}}$ is convergent to $\zeta$ uniquely and $\mathrm{CM} \alpha<1$, then $\zeta$ is a fixed point of $\mathrm{f}$. Moreover, if there exists another fixed point $\zeta^{*}$ such that $\sup _{\mathrm{n}} \mathrm{D}_{\mathrm{g}}\left(\mathrm{f}^{\mathrm{n}}\left(\mathrm{x}_{0}\right), \zeta^{*}\right)<\infty, \mathrm{n} \in \mathbb{N}$, then $\zeta^{*}=\zeta$.

Proof. We check the sequence $\left\{x_{n}\right\}_{n} \in \mathbb{N}$ for every $x \in O(x, f)$, it can be obtained that $\left\{x_{n}\right\}_{n} \in \mathbb{N}$ is a Cauchy sequence as proved in Theorem 4.3. Because $X$ is $D_{g}$-complete implies that $X$ is f-orbitally complete and there is always the orbit of $x$ for all $x \in X$, which implies the conditions of Theorem 4.3 are met, therefore $f$ has a fixed point $\zeta$ (see the proof of Theorem 4.3), so we need only prove that the fixed point of $f$ is unique. Suppose that $f$ has another fixed point $\zeta^{*}$ such that $\sup D_{g}\left(f^{n}\left(x_{0}\right), \zeta^{*}\right)<\infty$, then by $\left(\operatorname{RGM}_{3}\right)$,

$$
D_{g}\left(\zeta, \zeta^{*}\right) \leqslant C \varphi\left(\limsup _{n \rightarrow \infty} D_{g}\left(f^{n}\left(x_{0}\right), \zeta^{*}\right)\right)
$$

Because $\varphi(t)$ is continuous when $t \geqslant 0$ and $\sup _{n} D_{g}\left(f^{n}\left(x_{0}\right), \zeta^{*}\right)<\infty$, therefore

$$
\mathrm{C} \varphi\left(\lim _{n \rightarrow \infty} \sup _{n} D_{g}\left(f^{n}\left(x_{0}\right), \zeta^{*}\right)\right)<\infty,
$$

that is, $\mathrm{D}_{\mathrm{g}}\left(\zeta, \zeta^{*}\right)<\infty$. On the other hand,

$$
\begin{aligned}
D_{g}\left(\zeta, \zeta^{*}\right) & =D_{g}\left(f(\zeta), f\left(\zeta^{*}\right)\right) \\
& \leqslant \alpha \max \left\{D_{g}\left(\zeta, \zeta^{*}\right), D_{g}(\zeta, f(\zeta)), D_{g}\left(\zeta^{*}, f\left(\zeta^{*}\right)\right), \frac{1}{2}\left[D_{g}\left(\zeta, f\left(\zeta^{*}\right)\right)\right.\right.
\end{aligned}
$$




$$
\begin{aligned}
& \left.\left.+\mathrm{D}_{\mathrm{g}}\left(\zeta^{*}, f(\zeta)\right)\right]\right\}, \\
= & \alpha \max \left\{\mathrm{D}_{\mathrm{g}}\left(\zeta, \zeta^{*}\right), \mathrm{D}_{\mathrm{g}}(\zeta, \zeta), \mathrm{D}_{\mathrm{g}}\left(\zeta^{*}, \zeta^{*}\right)\right\} .
\end{aligned}
$$

Similarly, $\mathrm{D}_{g}(\zeta, \zeta) \leqslant \alpha \max \left\{\mathrm{D}_{\mathrm{g}}(\zeta, \zeta)\right\}=\alpha \mathrm{D}_{\mathrm{g}}(\zeta, \zeta)$, because sup $\mathrm{D}_{\mathrm{g}}\left(\mathrm{f}^{\mathrm{n}}(\mathrm{x}), \mathrm{x}\right)<\infty$, it is derived $\mathrm{D}_{\mathrm{g}}(\zeta, \zeta)=$ $D_{g}(\zeta, f(\zeta))<\infty$, hence $D_{g}(\zeta, \zeta)=0$ since $D_{g}(\zeta, \zeta) \leqslant \alpha D_{g}(\zeta, \zeta)$ and $1<\alpha<1$. In the same way, one can obtain $\mathrm{D}_{\mathrm{g}}\left(\zeta^{*}, \zeta^{*}\right)=0$, thus the inequality

$$
\mathrm{D}_{\mathrm{g}}\left(\zeta, \zeta^{*}\right) \leqslant \alpha \mathrm{D}_{\mathrm{g}}\left(\zeta, \zeta^{*}\right)
$$

holds. Consequently, $D_{g}\left(\zeta, \zeta^{*}\right)=D_{g}\left(f(\zeta), f\left(\zeta^{*}\right)\right) \leqslant \alpha D_{g}\left(\zeta, \zeta^{*}\right)<\infty$ which implies $D_{g}\left(\zeta, \zeta^{*}\right)=0$. Using the property $\left(\mathrm{RGM}_{1}\right)$, we obtain $\zeta^{*}=\zeta$.

\section{Acknowledgment}

Project supported by China Postdoctoral Science Foundation (Grant No. 2014M551168, No. 2016T90244) and Natural Science Foundation of Heilongjiang Province of China (Grant No. A201410).

\section{References}

[1] A. Ait Taleb, E. Hanebaly, A fixed point theorem and its application to integral equations in modular function spaces, Proc. Amer. Math. Soc., 128 (2000), 419-426. 1

[2] P. Chaipunya, Y. J. Cho, P. Kumam, Geraghty-type theorems in modular metric spaces with an application to partial differential equation, Adv. Difference Equ., 2012 (2012), 12 pages. 1

[3] V. V. Chistyakov, Modular metric spaces, I, Basic concepts, Nonlinear Anal., 72 (2010), 1-14. 2.2

[4] L. B. Ćirić, A generalization of Banach's contraction principle, Proc. Amer. Math. Soc., 45 (1974), 267-273. 1, 4

[5] L. B. Ćirić, D. Miheț, R. Saadati, Monotone generalized contractions in partially ordered probabilistic metric spaces, Topology Appl., 156 (2009), 2838-2844. 1

[6] L. B. Ćirić, J. S. Ume, Some common fixed point theorems for weakly compatible mappings, J. Math. Anal. Appl., 314 (2006), 488-499. 1

[7] S. Czerwik, Contraction mappings in b-metric spaces, Acta Math. Inform. Univ. Ostraviensis, 1 (1993), 5-11. 1, 2.2

[8] X.-M. Fan, Fixed point theorems for cyclic mappings in quasi-partial b-metric spaces, J. Nonlinear Sci. Appl., 9 (2016), 2175-2189. 1

[9] L. F. Guseman, Jr., Fixed point theorems for mappings with a contractive iterate at a point, Proc. Amer. Math. Soc., 26 (1970), 615-618. 4

[10] P. Hitzler, A. K. Seda, Dislocated topologies, J. Electr. Eng., 51 (2000), 3-7. 1, 2.2

[11] N. Hussain, D. Dorić, Z. Kadelburg, S. Radenović, Suzuki-type fixed point results in metric type spaces, Fixed Point Theory Appl., 2012 (2012), 12 pages. 1

[12] M. Jleli, B. Samet, A generalized metric space and related fixed point theorems, Fixed Point Theory Appl., 2015 (2015), 14 pages. $1,2.1,2.2,2.2,3.5,3,3$

[13] S. Koshi, T. Shimogaki, On F-norms of quasi-modular spaces, J. Fac. Sci. Hokkaido Univ. Ser. I, 15 (1961), 202-218. 1

[14] K. Kuaketa, P. Kumam, Fixed points of asymptotic pointwise contractions in modular spaces, Appl. Math. Lett., 24 (2011), 1795-1798.

[15] J. Musielak, Orlicz spaces and modular spaces, Lecture Notes in Mathematics, Springer-Verlag, Berlin, (1983)

[16] J. Musielak, W. Orlicz, On modular spaces, Studia Math., 18 (1959), 49-65. 1

[17] H. Nakano, Modulared semi-ordered linear spaces, Maruzen Co., Ltd., Tokyo, (1950) 1, 2.2

[18] J. J. Nieto, R. Rodríguez-López, Contractive mapping theorems in partially ordered sets and applications to ordinary differential equations, Order, 22 (2005), 223-239. 1

[19] J. J. Nieto, R. Rodríguez-López, Existence and uniqueness of fixed point in partially ordered sets and applications to ordinary differential equations, Acta Math. Sin. (Engl. Ser.), 23 (2007), 2205-2212. 1

[20] T. K. Pal, M. Maiti, Extensions of fixed point theorems of Rhoades and Ćirić, Proc. Amer. Math. Soc., 64 (1977), $283-286$. 4

[21] D. Panthi, K. Jha, G. Porru, A fixed point theorem in dislocated quasi-metric space, Amer. J. Math. Statist., 3 (2013), 153-156. 1

[22] S. Rezapour, R. H. Haghi, N. Shahzad, Some notes on fixed points of quasi-contraction maps, Appl. Math. Lett., 23 (2010), 498-502. 1 
[23] B. E. Rhoades, A comparison of various definitions of contractive mappings, Trans. Amer. Math. Soc., 226 (1977), 257290. 1

[24] B. E. Rhoades, Extensions of some fixed point theorems of Ćirić, Maiti, and Pal, Math. Sem. Notes Kobe Univ., 6 (1978), 41-46. 1

[25] Z.-G. Wang, H.-L. Li, Fixed point theorems and endpoint theorems for $(\alpha, \psi)$-Meir-Keeler-Khan multivalued mappings, Fixed Point Theory Appl., 2016 (2016), 18 pages. 1

[26] J. S. W. Wong, Two extensions of the Banach contraction mapping principle, J. Math. Anal. Appl., 22 (1968), 438-443. 4

[27] S. Yamamuro, On conjugate spaces of Nakano spaces, Trans. Amer. Math. Soc., 90 (1959), 291-311. 1

[28] F. M. Zeyada, G. H. Hassan, M. A. Ahmed, A generalization of a fixed point theorem due to Hitzler and Seda in dislocated quasi-metric spaces, Arab. J. Sci. Eng. Sect. A Sci., 31 (2006), 111-114. 1 